specific for congenital muscular dystrophy syndromes and histopathological support for the diagnosis. A finding of reduced merosin in muscle is predictive of severe weakness and disability.

Classical (Occidental) Merosin-positive form of CMD was milder and more slowly progressive than the merosin-negative form and Fukuyama type in a clinical and pathological study of 50 patients examined at the National Institute of Neuroscience, National Center of Neurology and Psychiatry, Kodaira, Tokyo, Japan. (Kobayashi O et al. Congenital muscular dystrophy: Clinical and pathological study of 50 patients with the classical (Occidental) merosin-positive form. Neurology March 1996;46:815-818).

Cognitive dysfunction in Becker's muscular dystrophy was the major presenting feature in 4 patients reported from the Children's Hospital, Boston, the Texas Children's Hospital, Houston, and other centers. (North KN, Miller G et al. Neurology March 1996;46:461-465). Psychiatric disturbance was also a feature in the absence of muscle weakness. An elevated serum creatine kinase may provide a valuable screening test in boys with unexplained cognitive or psychiatric disturbance. One patient had received various drugs used for ADHD before diagnosis was determined.

\title{
LEARNING DISABILITIES
}

\section{ARTICULATORY FEEDBACK DEFICIT IN DYSLEXIA}

An efferent or "motor-articulatory feedback" hypothesis for developmental dyslexia-phonological type is proposed from the Departments of Neurology and Psychiatry, University of Florida College of Medicine, Gainesville, FL. Most children learn to read by the alphabetic system, requiring phonological awareness and conversion of letters (graphemes) into speech sounds (phonemes). Most dyslexics have deficient phonological awareness and difficulty converting graphemes into phonemes. The left inferior frontal lobe is important in phonological reading, as suggested by patients with acquired lesions and PET studies of normal subjects. Dyslexic children are unable to perceive the position and movement of the articulatory apparatus (mouth, lips, tongue) during speech, impairing phonological awareness and conversion of graphemes to phonemes. Deficits in motorarticulatory programming or feedback may be related to this lack of awareness of articulators. (Heilman KM et al. Developmental dyslexia: a motorarticulatory feedback hypothesis. Ann Neurol March 1996:39:407-412). (Respond: Dr Kenneth M Heilman, Box 100236, University of Florida, Gainesville, FL 32610).

COMMENT. Other current hypotheses for developmental dyslexia are 1) visual hypothesis, with dysfunction in the visual perception system, and 2) auditory hypothesis, with abnormalities in the rapid discrimination of lowcontrast, complex sounds and associated speech and language disturbances.

A disconnection syndrome hypothesis for developmental dyslexia is proposed based on evidence from PET scanning studies conducted at the MRC Cognitive Development Unit, London, UK. (Paulesu E, Frith U et al. Brain Feb 1996;119:143-157). A rhyming and a short-term memory task with visually presented letters was used to study brain activity in 5 compensated adult developmental dyslexics. Brain regions normally activated in phonological processing were defective, and weak connections between anterior and posterior language areas are proposed. 